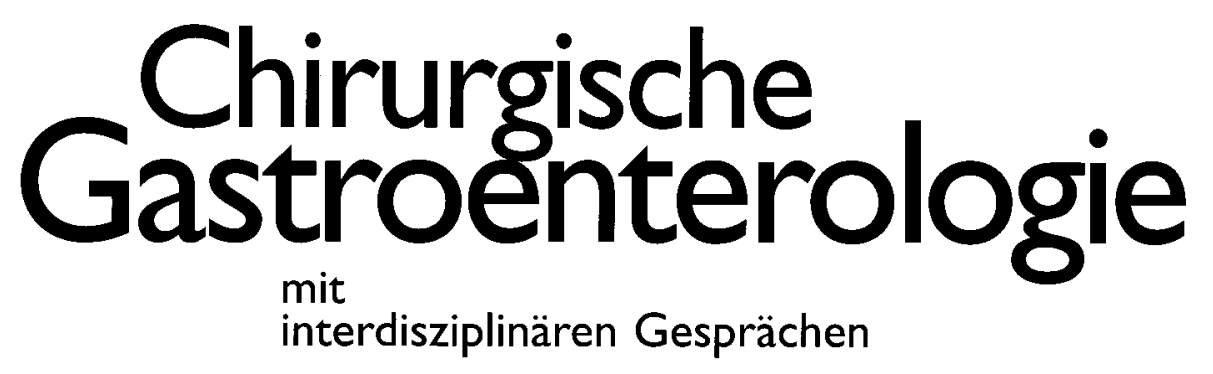

Hauptthema

J. F. Riemann,
Ludwigshafen
R. Bittner,
Stuttgart (Hrsg.)

\title{
Benigne und maligne Gallenwegstenosen
}

Eingeladene Beiträge

Interdisziplinäres Gespräch

Weitere Rubriken

Minimalinvasive Chirurgie

Prä- und postoperative Therapie

Buchbesprechungen

Tagungen und Kongresse 


\section{Gründungsherausgeber}

A. Akovbiantz $\dagger$, Zürich

H. Denck, Wien

K.-J. Paquet, Bad Kissingen

C. E. Zöckler, Bad Oeynhausen

\section{Hauptschriftleiter}

R. Bittner, Stuttgart

K.-J. Paquet, Hannover

\section{Schriftleitung}

Ch. E. Broelsch, Essen

M. W. Büchler, Bern

I. Gastinger, Cottbus

K. Glaser, Wien

J. Horn, München

J. R. Izbicki, Hamburg

E. Klar, Heidelberg

J. Mössner, Leipzig

H. Säuberli, Baden

R. Schiessel, Wien

\section{Wissenschaftlicher Beirat}

J. Alexander-Williams, Birmingham

H. M. Becker, München

M. Betzler, Essen

E. Bodner, Innsbruck

H. Bosseckert, Jena

H. Bülow, Schweinfurt

R. Dölp, Fulda

K. E. Frede, Basel

J. Gallinger, Moskau

A. Gangl, Wien

J. M. Hackl, Innsbruck

E. G. Hahn, Erlangen/Nürnberg

J. Hauss, Leipzig

J. M. Henderson, Cleveland

P. Hermanek, Erlangen/Nürnberg

R. A. Hinder, Omaha

W. Hohenberger, Erlangen/Nürnberg

Y. Idezuki, Tokyo

I. Ihse, Lund

C. W. Imrie, Glasgow

G. G. Jamieson, Adelaide

S. L. Jensen, Århus

K. Junghanns, Ludwigsburg

P. J. Kestens, Brüssel

B. Langer, Toronto

S. Liebe, Rostock

H. Lippert, Magdeburg
P. Malfertheiner, Magdeburg

K. Maruyama, Tokyo

Ch. Meyer, Straßburg

W. C. Meyers, Worcester

P. Morel, Genf

E. Moreno-Gonzáles, Madrid

V. Pegan, Ljubljana

F. M. Penninckx, Leuven

A. Peracchia, Mailand

R. Porschen, Tübingen

H. B. Reith, Würzburg

J. F. Riemann, Ludwigshafen

L. F. Rikkers, Madison

J. Rodés, Barcelona

H.-D. Saeger, Dresden

R. Schlumpf, Aarau

W.-H. Schmiegel, Bochum

J. Schölmerich, Regensburg

M. Stolte, Bayreuth

K. Sugimachi, Fukuoka

J. Terblanche, Kapstadt

M. Thelen, Mainz

A. Thiede, Würzburg

A. L. Warshaw, Boston

Ch. Wittekind, Leipzig

Wu Zaide, Wuhan

\title{
Bibliographische Dienste: \\ EMBASE/ Excerpta Medica \\ Reference Update
}

S. Karger $\cdot$ Medical and Scientific Publishers $\cdot$ Basel $\cdot$ Freiburg $\cdot$ Paris $\cdot$ London $\cdot$ New York $\cdot$ New Delhi $\cdot$ Bangkok $\cdot$ Singapore $\cdot$ Tokyo $\cdot$ Sydney

Die Zeitschrift erscheint dreimonatlich: pro Jahr erscheint 1 Band zu je 4 Heften. Bezugspreis für Jahrgang 15, 1999: DEM 220.- / SFr 172,-- einschließlich MwSt., zuzüglich Postgebühren. Der Abonnementpreis ist im voraus zahlbar. Das Abonnement der Zeitschrift läuft weiter, wenn es nicht spätestens 4 Wochen vor Abschluß eines Bandes abbestellt wird.

Abonnementbestellungen können bei jeder Buchhandlung oder direkt beim Verlag aufgegeben werden:

Bundesrepublik

Deutschland:

S. Karger GmbH,

Lörracher Str. 16 a,

D-79115 Freiburg,

Telefon (0761) 452070

Fax (07 61) 4520714 ,

\author{
Übrige Länder \\ S. Karger AG, \\ Allschwilerstr. 10, \\ Postfach, \\ CH-4009 Basel, \\ E-mail Karger@Karger.ch
}

Anzeigen: S. Karger

Verlag für Medizin und Naturwissenschaften $\mathrm{GmbH}$

Lörracher Str. 16a. D-79115 Freiburg, Tel. (07 61) 452070.

Gültig ist die Preisliste Nr. 7 von 1. Januar 1999.

Für den Inhalt außerhalb des redaktionellen Teiles (insbesondere Anzeigen, Industrieinformationen, Pressezitate und Kongreßinformationen) übernehmen Schriftleitung, Beirat und Verlag keine Gewähr.

Eine Markenbezeichnung kann warenzeichenrechtlich geschützt sein, auch wenn bei ihrer Verwendung in dieser Zeitschrift das Zeichen ${ }^{\circledR}$ oder ein anderer Hinweis auf etwa bestehende Schutzrechte fehlen sollte. Für Satzfehler, insbesondere bei Dosierungsangaben, wird keine Gewähr übernommen.

Die Zeitschrift sowie alle in ihr enthaltenen einzelnen Beiträge und Abbildungen sind urheberrechtlich geschützt. Jede Verwertung, die nicht ausdrücklich vom Urheberrechtsgesetz zu- gelassen ist, bedarf der vorherigen Zustimmung des Verlags. Das gilt insbesondere für Vervielfältigung, Bearbeitungen, Übersetzungen, Mikroverfilmungen und die Einspeicherung und Verarbeitung in elektronischen Systemen.

두 Copyright 1999 by S. Karger

Verlag für Medizin und Naturwissenschaften $\mathrm{GmbH}$ Lörracher Straße 16 a, D-79115 Freiburg

Verlagsleitung und presserechtlich verantwortlich: Sibylle Hopf

Redaktionsassistenz: Dr. Hannelore Tenckhoff

Anzeigenleitung: Susanne Meister

Produktionsleitung: Georg Brunner

Herstellung: druckhaus köthen $\mathrm{GmbH}$

Friedrichstraße 11/12, D-06366 Köthen/Anhalt

ISBN 3-8055-6829-0

\section{KARGER}

Fax +497614520714

E-mailkargergmbh@aol.com

www.karger.com 
Editorial

6 Benigne und maligne Gallenwegerkrankungen

Riemann, J. F. (Ludwigshafen); Bittner, R. (Stuttgart)

Hauptthema

7 Cholestase: Definition, Differentialdiagnose und laborchemische Diagnostik

Moradpour, D.; Schwacha, H.; Allgaier, H.-P.; Blum, H. E. (Freiburg in Br.)

12 Endoskopische Diagnostik und interventionelle Therapie bei benignen Gallengangstenosen

Schilling, D.; Jakobs, R.; Adamek, H. E.; Riemann, J. F. (Ludwigshafen)

20 Intraduktale Endoskopie und Endosonographie des

Gallengangsystems

Seifert, H.; Schmitt, T.; Wehrmann, T. (Frankfurt/M.)

28 Nichtinvasive Diagnostik von Gallenwegstenosen mit der Magnetresonanz-Cholangiopankreatikographie

König, C. W.; Duda, St. H.; Pinocy, J.; Gottwald, Th.; Trübenbach, J.;

Müller-Schimpfle, M.; Claussen, C. D. (Tübingen)

34 Benigne Gallengangstrikturen: Wie effektiv ist die chirurgische Therapie? Eine retrospektive Analyse über einen Zeitraum von 26 Jahren

Paquet, K. J. (Bad Kissingen)

40 Lokalisations- und stadienabhängige Therapie der KlatskinTumoren

Klempnauer, J.; Schrem, H. (Bochum)

48 Operative Strategie bei periampullärem Karzinom und Ikterus

Z'graggen, K.; Kulli, Ch.; Holzinger, F.; Friess, H.; Büchler, M. W. (Bern)

55 Cholestase bei chronischer Pankreatitis: Ergebnisse der modifizierten duodenumerhaltenden

Pankreaskopfresektion

Schlosser, W.; Beger, H.-G. (Ulm)
Editorial

6 Benign and Malignant Cholepathias

Riemann, J. F. (Ludwigshafen); Bittner, R. (Stuttgart)

Main Theme

7 Cholestasis: Definition, Differential Diagnosis and Laboratory Diagnostics

Moradpour, D.; Schwacha, H.; Allgaier, H.-P.; Blum, H. E. (Freiburg in Br.)

12 Endoscopics Diagnostics and Interventional Therapy in Patients with Bile Duct Stenoses

Schilling, D.; Jakobs, R.; Adamek, H. E.; Riemann, J. F. (Ludwigshafen)

20 Intraductal Endoscopy and Endosonography of Bile Ducts

Seifert, H.; Schmitt, T.; Wehrmann, T. (Frankfurt/M.)

28 Noninvasive Diagnostics of Biliary Stenosis with Magnetic Resonance Cholangiopancreatography

König, C. W., Duda, St. H.; Pinocy, J.; Gottwald, Th.; Trübenbach, J.; Müller-Schimpfle, M.; Claussen, C. D. (Tübingen)

34 Benign Biliary Stricture: How Effective is the Surgical Therapy? A Retrospective Analysis over a Period of 26 Years

Paquet, K. J. (Bad Kissingen)

40 Therapy of Klatskin Tumours in Relation to Their Localisation and Tumour Stage Klempnauer, J.; Schrem, H. (Bochum)

48 Periampullary Carcinoma and Jaundice: Surgical Strategy Z'graggen, K.; Kulli, Ch.; Holzinger, F.; Friess, H.; Büchler, M. W. (Bern)

55 Cholestasis in Patients with Chronic Pancreatitis: Results of the Modified Duodenum-Preserving Pancreatic Head Resection Schlosser, W.; Beger, H.-G. (Ulm)

\section{KARGER}

Fax +497614520714

E-mailkargergmbh@aol.com

www.karger.com (c) 1999 S. Karger GmbH, Freiburg

Artikel (Volltext) und Inhaltsverzeichnisse des nächsten Heftes: www.karger.com/journals/cga/cga_bk.htm 
62 Verschlußikterus - palliative Drainage aus der Sicht des Endoskopikers

Arnold, J. C.; Riemann, J. F. (Ludwigshafen)

68 Verschlußikterus - palliative Drainage aus der Sicht des Radiologen

Duda, St. H., König, C. W.; Pinocy, J.; Schott, U.; Pereira, Ph.; Huppert, P. E.; Viebahn, R.; Claussen, C. D. (Tübingen)

73 Verschlußikterus - palliative Drainage aus der Sicht des Chirurgen

Manger, Th.; Pross, M.; Lippert, H. (Magdeburg)

78 Photodynamische Therapie bei malignen Gallengangtumoren Erfahrungen und Perspektiven

Ortner, M.-A. E. J. (Berlin)

Interdisziplinäres Gespräch

82 Interventionelle Therapie benigner und maligner

Gallenwegstenosen

Neuhaus, H. (Düsseldorf) (Gesprächsleiter)

Minimalinvasive Chirurgie

88 Mirizzi-Syndrom: Kontraindikation für die laparoskopische Operation?

Ulrich, M.; Nick, G.; Bittner, R. (Stuttgart)

Prä- und postoperative Therapie

92 Medikamentöse Behandlung der akuten Ösophagus- und Magenvarizenblutung

Borroughs, A. K. (London)

100 Buchbesprechungen

102 Tagungen und Kongresse

2 Impressum

104 Richtlinien für Autoren
62 Obstructive Jaundice - Palliative Drainage from an Endoscopist's Point of View

Arnold, J.C.; Riemann, J.F. (Ludwigshafen)

68 Obstructive Jaundice - Palliative Drainage from a Radiologist's Point of View

Duda, St. H., König, C.W.; Pinocy, J.; Schott, U.; Pereira, Ph.; Huppert, P. E.; Viebahn, R.; Claussen, C. D. (Tübingen)

73 Obstructive Jaundice - Palliative Drainage from a Surgeon's Point of View

Manger, Th.; Pross, M.; Lippert, H. (Magdeburg)

78 Photodynamic Therapy in Cholangioma - First Experiences and Perspectives Ortner, M.-A. E. J. (Berlin)

Interdisciplinary Discussion

82 Interventional Therapy of Benign and Malignant Stenoses of Bile Ducts

Neuhaus, H. (Düsseldorf) (Gesprächsleiter)

Minimally Invasive Surgery

88 Mirizzi Syndrome: Contraindication for Laparoscopic Operation?

Ulrich, M.; Nick, G.; Bittner, R. (Stuttgart)

Pre- and Postoperative Therapy

92 Medical Treatment of Acute Variceal Bleeding

Borroughs, A. K. (London)

100 Book Reviews

102 Meetings and Conferences

2 Imprint

U3 Instructions for the Authors

\section{KARGER}

Fax +497614520714

E-mailkargergmbh@aol.com

www.karger.com (c) 1999 S. Karger GmbH, Freiburg

Artikel (Volltext) und Inhaltsverzeichnisse des nächsten Heftes: www.karger.com/journals/cga/cga_bk.htm 


\title{
In Vorbereitung
}

\begin{abstract}
Antibiotikaprophylaxe in der Gallenwegchirurgie
\end{abstract}
Wittmann, D. H. (Wisconsin)

Hepatikolithiasis - endoskopisch-interventionelle Therapiemöglichkeiten

Jakobs, R.; Riemann, J. F. (Ludwigshafen)

Primär sklerosierende Cholangitis - Therapieoptionen

Maier, K. P. (Tübingen)

Indikation und Ergebnisse der Lebertransplantation bei primär sklerosierender Cholangitis Otto, G. (Mainz)

\section{Ösophaguskarzinom}

Petrasch, S. (Bottrop)

Leiomyosarkom der Leber und Vena-cava-Kompressionssyndrom bei einer 66jährigen Patientin - eine Kasuistik

Kahlenberg, D.; Will, U.; Scheele, J.; Werner, B.; Katenkamp, D.; Bosseckert, H. (Jena)

Subklinische portosystemische Enzephalopathie und Lungenstrombahnveränderungen bei Kindern und Jugendlichen mit portaler Hypertension bei prähepatischem Block Otting, U.; Hellmann, C.; Planke, C.; Lehmann, R.; Gottschalk, S. (Berlin) 